\title{
Synovial Fluid Progenitors Expressing CD90+ from Normal but Not Osteoarthritic Joints Undergo Chondrogenic Differentiation without Micro-Mass Culture
}

\author{
Roman J. Krawetz ${ }^{1,2 *}$, Yiru Elizabeth $\mathrm{Wu}^{1}$, Liam Martin ${ }^{3}$, Jerome B. Rattner ${ }^{2}$, John R. Matyas ${ }^{4}$, \\ David A. Hart' ${ }^{1}$
}

1 Department of Surgery, University of Calgary, Calgary, Alberta, Canada, 2 Department of Cell Biology and Anatomy, University of Calgary, Calgary, Alberta, Canada, 3 Department of Medicine, University of Calgary, Calgary, Alberta, Canada, 4 Department of Comparative Biology and Experimental Medicine, Faculty of Veterinary Medicine, University of Calgary, Calgary, Alberta, Canada

\begin{abstract}
Objective: Mesenchymal progenitor cells (MPCs) can differentiate into osteoblasts, adipocytes, and chondrocytes, and are in part responsible for maintaining tissue integrity. Recently, a progenitor cell population has been found within the synovial fluid that shares many similarities with bone marrow MPCs. These synovial fluid MPCs (sfMPCs) share the ability to differentiate into bone and fat, with a bias for cartilage differentiation. In this study, sfMPCs were isolated from human and canine synovial fluid collected from normal individuals and those with osteoarthritis (human: clinician-diagnosed, canine: experimental) to compare the differentiation potential of CD90+ vs. CD90- sfMPCs, and to determine if CD90 (Thy-1) is a predictive marker of synovial fluid progenitors with chondrogenic capacity in vitro.

Methods: sfMPCs were derived from synovial fluid from normal and OA knee joints. These cells were induced to differentiate into chondrocytes and analyzed using quantitative PCR, immunofluorescence, and electron microscopy.

Results: The CD90+ subpopulation of sfMPCs had increased chondrogenic potential compared to the CD90- population. Furthermore, sfMPCs derived from healthy joints did not require a micro-mass step for efficient chondrogenesis. Whereas sfMPCs from OA synovial fluid retain the ability to undergo chondrogenic differentiation, they require micro-mass culture conditions.

Conclusions: Overall, this study has demonstrated an increased chondrogenic potential within the CD90+ fraction of human and canine sfMPCs and that this population of cells derived from healthy normal joints do not require a micro-mass step for efficient chondrogenesis, while sfMPCs obtained from OA knee joints do not differentiate efficiently into chondrocytes without the micro-mass procedure. These results reveal a fundamental shift in the chondrogenic ability of cells isolated from arthritic joint fluids, and we speculate that the mechanism behind this change of cell behavior is exposure to the altered milieu of the OA joint fluid, which will be examined in further studies.
\end{abstract}

Citation: Krawetz RJ, Wu YE, Martin L, Rattner JB, Matyas JR, et al. (2012) Synovial Fluid Progenitors Expressing CD90+ from Normal but Not Osteoarthritic Joints Undergo Chondrogenic Differentiation without Micro-Mass Culture. PLoS ONE 7(8): e43616. doi:10.1371/journal.pone.0043616

Editor: Irina Kerkis, Instituto Butantan, Brazil

Received February 7, 2012; Accepted July 23, 2012; Published August 29, 2012

This is an open-access article, free of all copyright, and may be freely reproduced, distributed, transmitted, modified, built upon, or otherwise used by anyone for any lawful purpose. The work is made available under the Creative Commons CCO public domain dedication.

Funding: This study was supported by research grants from Pfizer, Alberta Innovates-Health Solutions team grant in osteoarthritis, and Canadian Institutes for Health Research (MOP 79384). The funders had no role in study design, data collection and analysis, decision to publish, or preparation of the manuscript.

Competing Interests: The authors have the following interest: This study was partly funded by Pfizer Inc. There are no patents, products in development or marketed products to declare. This does not alter the authors' adherence to all the PLOS ONE policies on sharing data and materials, as detailed online in the guide for authors.

*E-mail: rkrawetz@ucalgary.ca

\section{Introduction}

Within the last decade, the synovium and synovial fluid have been identified as a source of mesenchymal progenitor/stem cells (sMPCs) that are functionally distinct from bone marrow MPCs [1-7]. Furthermore, these synovial membrane (smMPCs) and sfMPCs can assist in cartilage repair both in vivo and in vitro [8-11]. There is compelling evidence that these cells can be used effectively to promote cartilage repair [1-11]. sfMPCs reportedly have increased chondrogenic potential $[12,13]$ compared to bone marrow-derived mesenchymal stem cells (bmMPCs), including increased expression of the hyaluronan receptor CD44 [13,14] and uridine diphosphoglucose dehydrogenase (UDPGD), an enzyme required for hyaluronan synthesis. Notably, bmMPCs and other MPG populations do not express UDPGD [13,14]. Evidence from in vivo studies have demonstrated that when partialthickness defects in the articular cartilage of rabbits are formed, a continuous cell layer extending from the synovial membrane is observed to contribute to the repair of the cartilage either with or without chondrogenic inducers present $[9,10]$. In addition, pig and 
human sfMPCs have been transformed in vitro into scaffolds termed Tissue Engineering Constructs (TECs) that can be used to repair cartilaginous defects (in pigs) in vivo [6,7]. Additionally, another group has recently demonstrated that smMPCs have the ability to bind directly to cartilage ex vivo in minutes, and are able to contribute to cartilage repair in a defect model [11]. Human sfMPCs are typically characterized using cluster of differentiation (CD) antigens [15]: CD105 (Endoglin), CD90 (Thy-1), CD73 (Ecto-5'-nucleotidase) and CD44 are present on the surface of MPCs/MSCs, while CD45 (Protein tyrosine phosphatase, receptor type, $\mathrm{C}$ ) and $\mathrm{CD} 1 \mathrm{lb}$ (Integrin alpha $\mathrm{M}$ ) are not expressed by this cell population [15]. The present study focuses on CD90 (Thy1), which has been shown to interact with Integrins, tyrosine kinases, growth factors, and cytokines thereby promoting downstream cellular events including: adhesion, apoptosis, proliferation, and migration [16]. CD90 is commonly used as a marker of MPCs/MSCs, though it is also expressed by many other cell types including neurons, endothelial cells, T-cells, and other immune/ non-immune cell types [16]. More recently, CD90 has been utilized as a selection marker of multi-potent progenitors from bone marrow, synovial tissues, fat, amnion and other tissues [17]. However, the exact role of CD90 on the surface of this class of cells remains unknown.

A number of recent studies have begun to explore the role of sfMPCs in diseases, including arthritis. Initial reports suggested that there was no difference in the chondrogenic potential of sfMPCs derived from healthy joints and joints with osteoarthritis (OA) or rheumatoid arthritis (RA) [2], notwithstanding the increase in number of sfMPCs in the OA knees [2]. A more recent study by the same group reported that the inflammatory intra-articular environment in RA joints is responsible for the reduced chondrogenic potential of sfMPCs [18]. As OA is generally viewed primarily as a degenerative rather than an inflammatory joint disease, it seems that the milieu of RA and OA joints has a fundamentally different influence on the capacity of sfMPCs to proliferate and differentiate. If, as has been speculated, sfMPCs participate in processes of joint maintenance or repair after injury $[9,10]$, a fuller understanding of sfMPGs is warranted as they are potential therapeutic targets for these common and debilitating joint diseases.

In a recent study where synovial membrane stem cells were obtained from OA patients and differentiated using a micro-mass tissue culture, a significant positive correlation was observed between CD90 expression and chondrogenic differentiation [19]. Therefore, the aim of the present study is a comparison of the chondrogenic potential of sfMPCs (human and canine) isolated from normal and osteoarthritic synovial fluid.

\section{Results}

\section{Differentiation potential of normal and OA derived sfMPCs}

To evaluate the chondrogenic potential of human sfMPCs (CD105+, CD73+, CD44+, CD45-. CD11b-) and canine sfMPCs (CD45-, CD34-) derived from normal and OA synovial fluid, the cells were differentiated into chondrocytes with media supplements over a 14 day period with a prior micro-mass aggregation step. At days 0, 3, 5, 8 and 14, mRNA was collected and probed using qRT-PGR for Sox9, Collagen 2, and Aggrecan (Figure 1 A,E,H,L). By day 14, Sox9, Collagen 2 and Aggrecan were significantly elevated compared to day 0 controls in normal (Figure $1 \mathrm{~A}, \mathrm{E}$ ) and OA (Figure $1 \mathrm{H}, \mathrm{L}$ ) sfMPCs derived from human and canine synovial fluid. Immunofluorescence confirmed the qRT-PCR data using a Collagen 2 antibody on day 14 .
sfMPC-derived chondrogenic masses from normal (Figure $1 \mathrm{~B}, \mathrm{~F}$ ) and OA (Figure 1 I,M) fluid expressed Collagen 2 protein on day 14. Secondary antibody alone controls demonstrated minimal non-specific staining in human (Figure $1 \mathrm{C}$ ) and canine (Figure $1 \mathrm{~J}$ ) sfMPCs. Furthermore, the micro-masses generated from all conditions stained with Alcian blue (Figure 1 D,G,K,N).

\section{Enhanced Chondrogenic Differentiation of CD90+ sfMPCs}

Since the human and canine sfMPCs contained CD90 positive and negative cells, the chondrogenic potential of the CD90positive and CD90-negative fractions within the sfMPC population were studied. Previously isolated sfMPCs (Figure 2) were further enriched for CD90 using immuno-magnetic separation, with the resultant CD90-positive and CD90-negative fractions induced to differentiate into chondrocytes utilizing a micro-mass step (Figure 2). CD90+ sfMPCs from normal individuals (human and canine) behaved in a similar fashion to the total sfMPC population, displaying significantly increased levels of Sox9, type II Collagen, and Aggrecan mRNA (Figure $2 \mathrm{~A}, \mathrm{~K}$ ) and demonstrated positive staining for Alcian blue (Figure 2 B,L) and Collagen 2 (Figure $2 \mathrm{C}, \mathrm{M}$ ) by day 14. The CD90-negative fraction displayed reduced levels of Sox9, Collagen 2 and Aggrecan mRNA (although significantly increased compared to day 0)(Figure 2 $\mathrm{A}, \mathrm{K}$ ), as well as less intense alcian blue (Figure $2 \mathrm{D}, \mathrm{N}$ ) and Collagen 2 (Figure $2 \mathrm{E}, \mathrm{O}$ ) staining at day 14 of differentiation compared to the CD90+ population. Similarly, human and canine CD90+ sfMPGs from OA fluid demonstrated increased Sox9, Collagen 2 and Aggrecan mRNA expression (Figure 2 F,P), as well as staining with alcian blue (Figure 2 G,Q) and Collagen 2 (Figure $2 \mathrm{H}, \mathrm{R}$ ) compared to the CD90- sfMPCs. Specifically, the mRNA level (Figure 2 F,P) and staining with alcian blue (Figure 2 $\mathrm{I}, \mathrm{S}$ ) and Collagen 2 (Figure $2 \mathrm{~J}, \mathrm{~T}$ ) were decreased in the CD90sfMPCs compared to the CD90+ sfMPGs.

To examine if there were any differences in the osteogenic or adipogenic capacity of CD90+/- human sfMPCs (normal \& OA), the cells were placed in adipogenic or osteogenic conditions and allowed to differentiate for 20 days (Figure 3). After 20 days of differentiation, the cells were stained for Alkaline phosphatase (Figure $3 \mathrm{~A}$ ) or Oil Red-O (Figure $3 \mathrm{~B}$ ), and assayed using qPCR for Adiponectin, PPAR-gamma, Osteonectin and Osterix (Sp7) (Figure $3 \mathrm{C}-\mathrm{F}$ ). Using these outcome measures, no differences in osteogenic or adipogenic differentiation capacity were observed between normal and OA sfMPGs, or CD90+/- sub-populations of either group.

\section{Chondrogenesis of normal CD90-positive sfMPCs without a prior micro-mass step}

Based on the foregoing results, the chondrogenic potential of normal and OA sfMPSc were studied without micro-mass culture. Without a prior micro-mass step, normal sfMPCs from human (Figure $4 \mathrm{~A}$ ) and canine (Figure 4 D) up-regulated Sox 9, Collagen 2 and Aggrecan mRNA during differentiation. In contrast, the Sox 9, Collagen 2 and Aggrecan mNRA levels in OA sfMPCs were significantly lower (Figure 4 A,D). Normal (human and canine) sfMPCs aggregated into small tissue-like structures during differentiation (Figure 4 B,G); OA-derived sfMPGs (human and canine) did not aggregate (Figure $4 \mathrm{C}, \mathrm{F}$ ).

\section{CD90-positive Cells Form a Tissue-like Structure}

The tissue-like structures formed by normal CD90+ sfMPCs stained positively with antibodies against Aggrecan (Figure $5 \mathrm{~A}$ ) and Collagen 2 (Figure $5 \mathrm{C}$ ). When visualized with TEM, these 


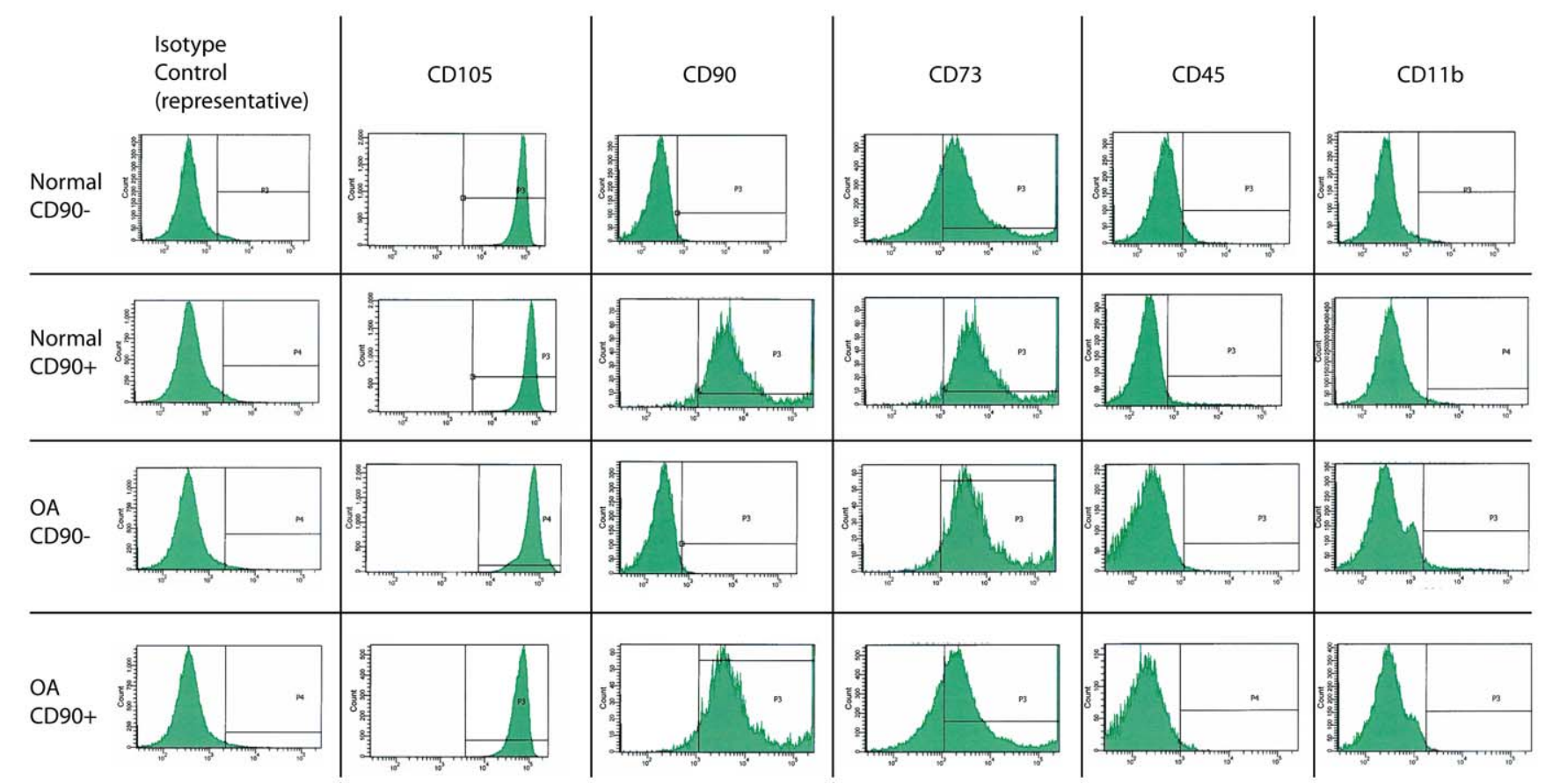

Figure 1. Micro-mass differentiation of sfMPCs. Normal (human $N=5$ A-D \& canine $N=2 \mathrm{H}-\mathrm{K}$ ) and $\mathrm{OA}$ (human $\mathrm{N}=5 \mathrm{E}-\mathrm{G}$ \& canine $\mathrm{N}=2 \mathrm{~L}-\mathrm{N}$ ) derived sfMPCs where aggregated using the micro-mass technique and induced to differentiate over a 14 day period with media supplements. qRTPCR results demonstrated that Sox9, Collagen 2 and Aggrecan are significantly elevated at days $3,5,8$ and 14 of differentiation $(A, E, H, L)$, furthermore, at day 14 Collagen 2 protein is expressed in the cell cultures $(B, F, I, M)$. Secondary controls $(C, J)$ demonstrate minimal non-specific staining. Scale bars represent $50 \mu \mathrm{M} .{ }^{*}=\mathrm{p}>0.05$.

doi:10.1371/journal.pone.0043616.g001

tissue-like structures were highly cellular (Figure 5 E). TEM analysis demonstrated that the cells were in close association with each other (Figure $5 \mathrm{~F}$ ), and were also in proximity to collagen fibres (Figure $5 \mathrm{G}$ ). The cells in these self-assembled structures were attached to the surrounding collagen fibre network of the extracellular matrix (Figure $5 \mathrm{E}$ and F).

\section{Discussion}

OA is a degenerative disorder of synovial joints characterized by articular cartilage degradation and meager evidence of repair or regeneration. Although evidence of cartilage stem/progenitor cells has been difficult to obtain, which may explain this response, recent animal studies have raised the possibility that MPCs present in the synovial membrane or joint fluid may play a role in repair cartilage in vivo $[9,10]$.

MPCs are of particular interest to regenerative medicine as they have the ability to generate bone, cartilage, and muscle under certain conditions. Indeed, properly harnessed, these MPGs could be utilized in-vivo to promote the repair or replacement of tissue damaged by trauma or disease. MPCs for skeletal regeneration are most commonly sourced from the bone marrow or fat, and it is increasing recognized that many adult tissues have resident stem cell populations. Although seemingly the ideal source of MPCs for cartilage regeneration, it has been claimed that adult articular cartilage uniquely lacks 'true' MPCs [21]. Interestingly, MPC populations have been isolated from the synovial membrane and synovial fluid bathing the joint [14]. Moreover, when compared to MPG populations isolated from bone marrow or fat [12], sfMPCs demonstrate an increased chondrogenic capacity compared to MPC populations isolated from bone marrow or fat [12].
This current study demonstrates that normal and OA (human and canine) derived sfMPCs undergo effective chondrogenesis when a micro-mass step is included in the differentiation protocol, which corroborates previously published findings [2]. Furthermore, when sfMPCs (normal/OA) are enriched for the CD90positive fraction, enhanced chondrogenesis is observed compared to the CD90-depleted fraction. This is of particular interest as a recent study by Nagase et al. reports that the chondrogenic potential of adult stem cells decrease with the subsequent loss of CD90 [19], while another study seems to contradict this observation [22]. The results of the present study supports those of Nagase et al. suggesting that CD90 is a cell surface marker that correlates positively with chondrogenic potential [19]. It is also noteworthy that patients with even advanced OA have sfMPCs, and in some cases with numbers far exceeding normal individuals [2], which could be interpreted as a cellular repair response.

A particularly noteworthy finding in the present study is that CD90-positive sfMPCs (normal/OA) can differentiate into chondrocytes without a micro-mass step. However, whereas whole populations of sfMPCs from normal individuals aggregate and express high levels of chondrogenic markers, whole populations of sfMPCs from OA joints do not aggregate spontaneously, grow only as a monolayer, and express low levels of chondrogenic markers during differentiation. The exact functional significance of micromass aggregation is unclear, yet micro-mass-induced aggregation clearly increases the efficiency of chondrogenesis [23,24], possibly by mimicking embryonic tissue condensation. Although the promotion of cell differentiation by aggregation is not limited to sfMPCs and chondrogenesis, (having been demonstrated with pancreatic, hepatic, fibroblastic, bone marrow, and cord blood MPGs [25-30]), it seems curious that all normal and OA sfMPCs behave differently in non-aggregating culture even though they 
A Normal human sMPCs

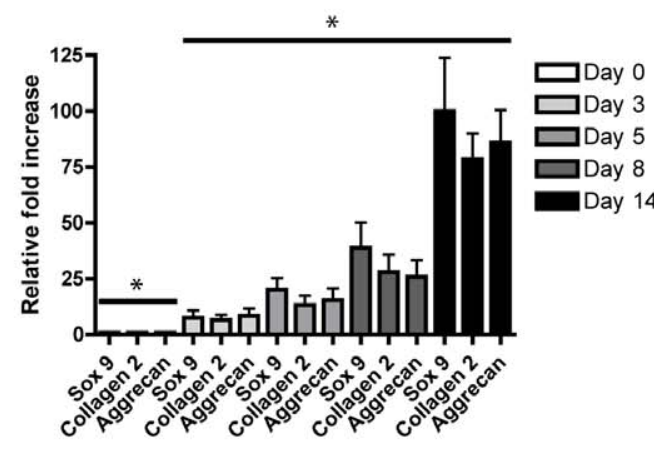

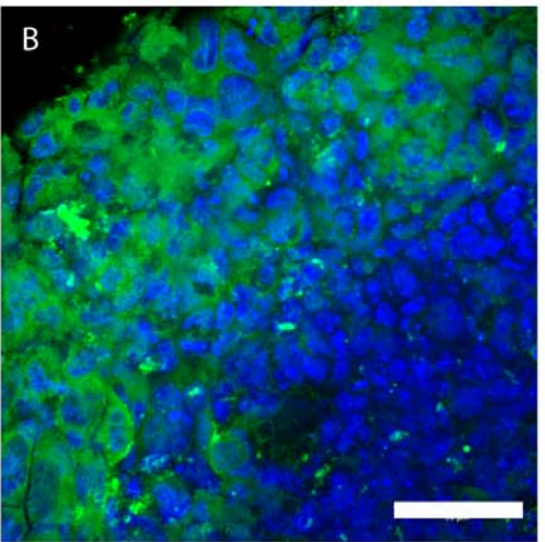
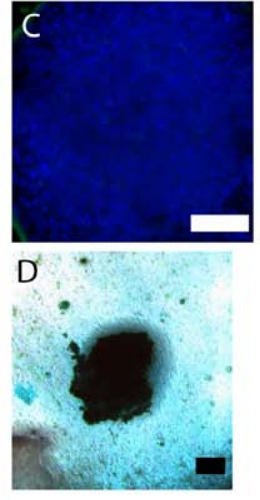

E Osteoarthritic human SMPCs

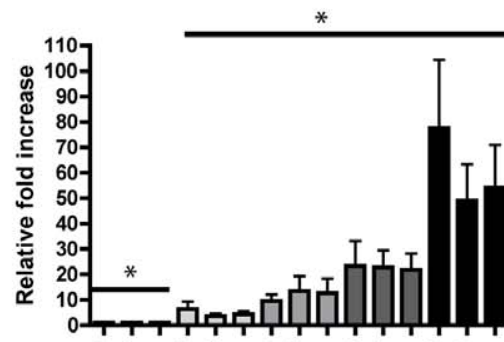

$92 \times 92 \times 92 \times 92=92$

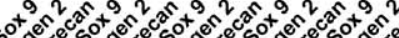

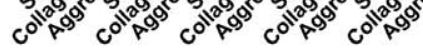

$\square$ Day 0 $\square$ Day 3

$\square$ Day 5

Day 8

Day 14
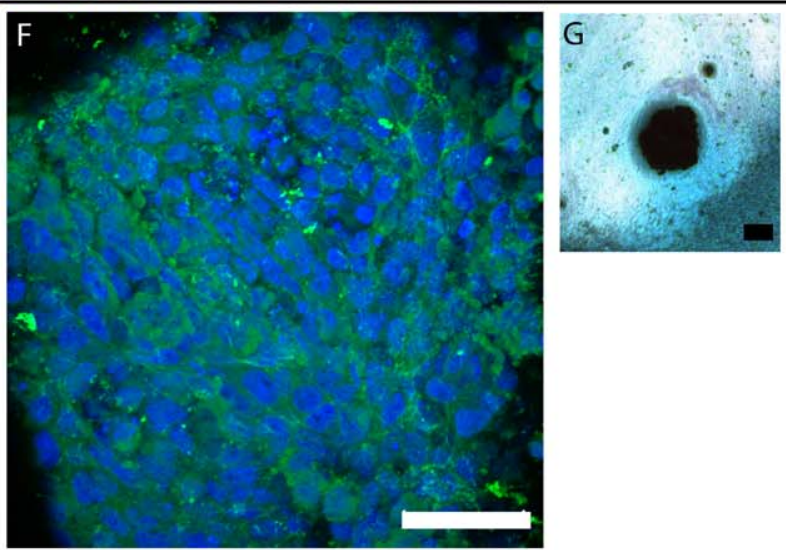

$\mathrm{H}$

Normal canine sMPCs
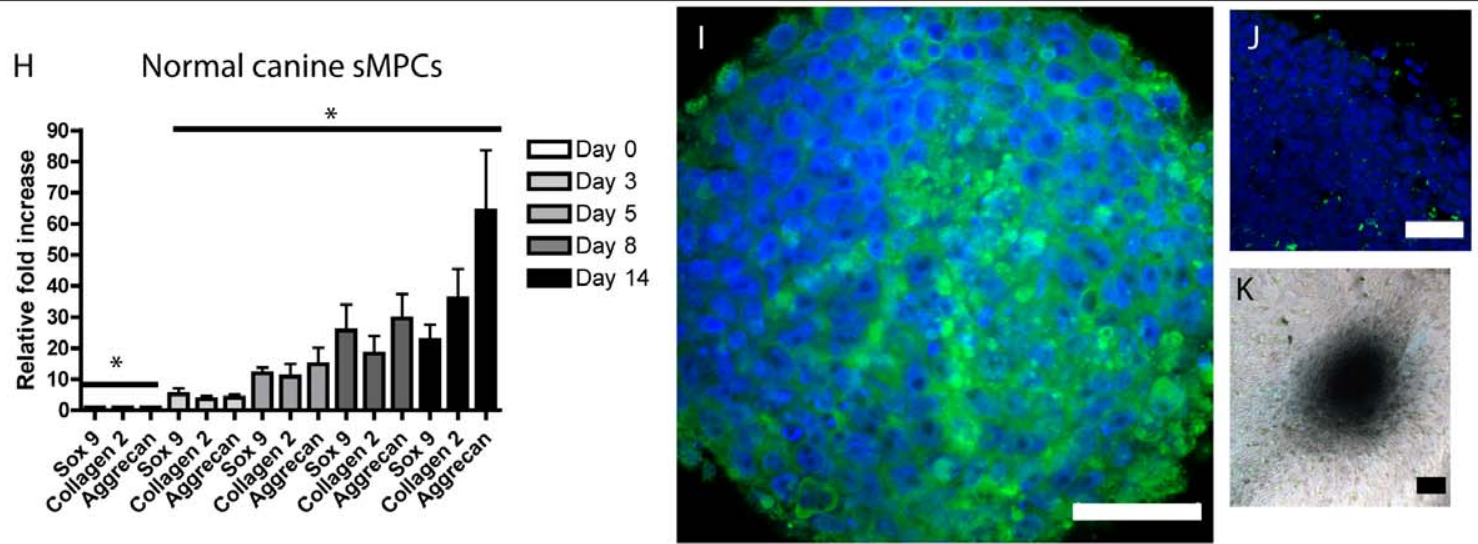

L Osteoarthritic canine sMPCs

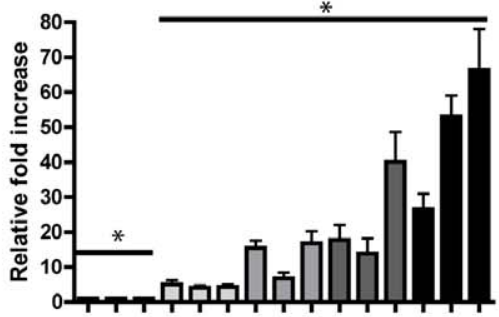

$\square$ Day 0

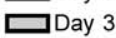

$\square$ Day 5

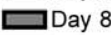

Day 14
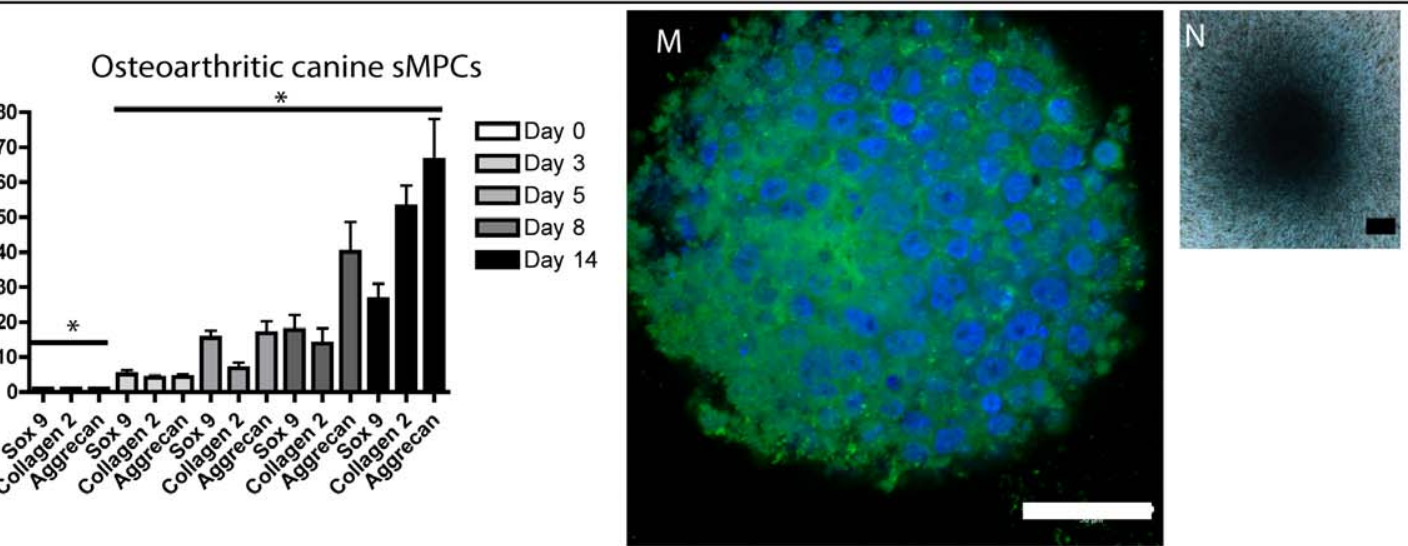

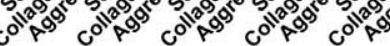

Figure 2. Chondrogenic Differentiation of CD90+/- sfMPCs. sfMPCs derived from normal synovial fluid were enriched/depleted for CD90 and induced to differentiate into chondrocytes. The CD90+ fraction expressed $(A, F, K, P)$ similar levels of Sox9, Collagen 2 and Aggrecan mRNA on day 14 to the total SMPC population (Figure 2), whereas the CD90- fraction demonstrated reduced levels of Sox9, Collagen 2 and Aggrecan (A,F,K,P). Alcian 
blue $(D, I, N, S)$ and Collagen $2(E, J, O, T)$ staining intensity was also decreased in the CD90- negative fraction compared to the CD90+ fraction (Alcian blue: $B, G, L, Q$, Collagen $2: C, H, M, R$ ) on day 14 . Scale bars represent $250 \mu \mathrm{M}$.

doi:10.1371/journal.pone.0043616.g002

A

Normal human
MPCs

Osteoarthritic
CD90+ MPCs

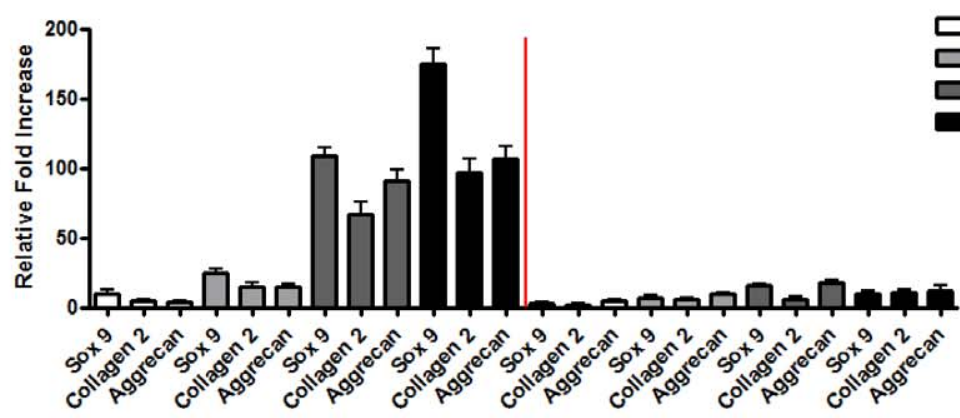

CD90+ MPCs

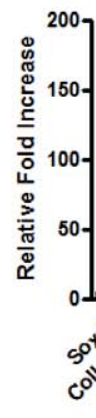

CD90-MPCs
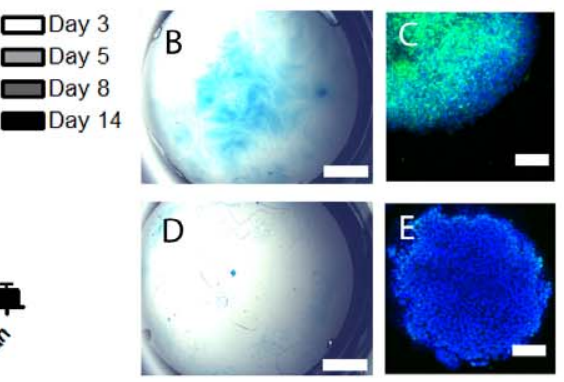

K

CD90+ MPCs

CD90- MPCs
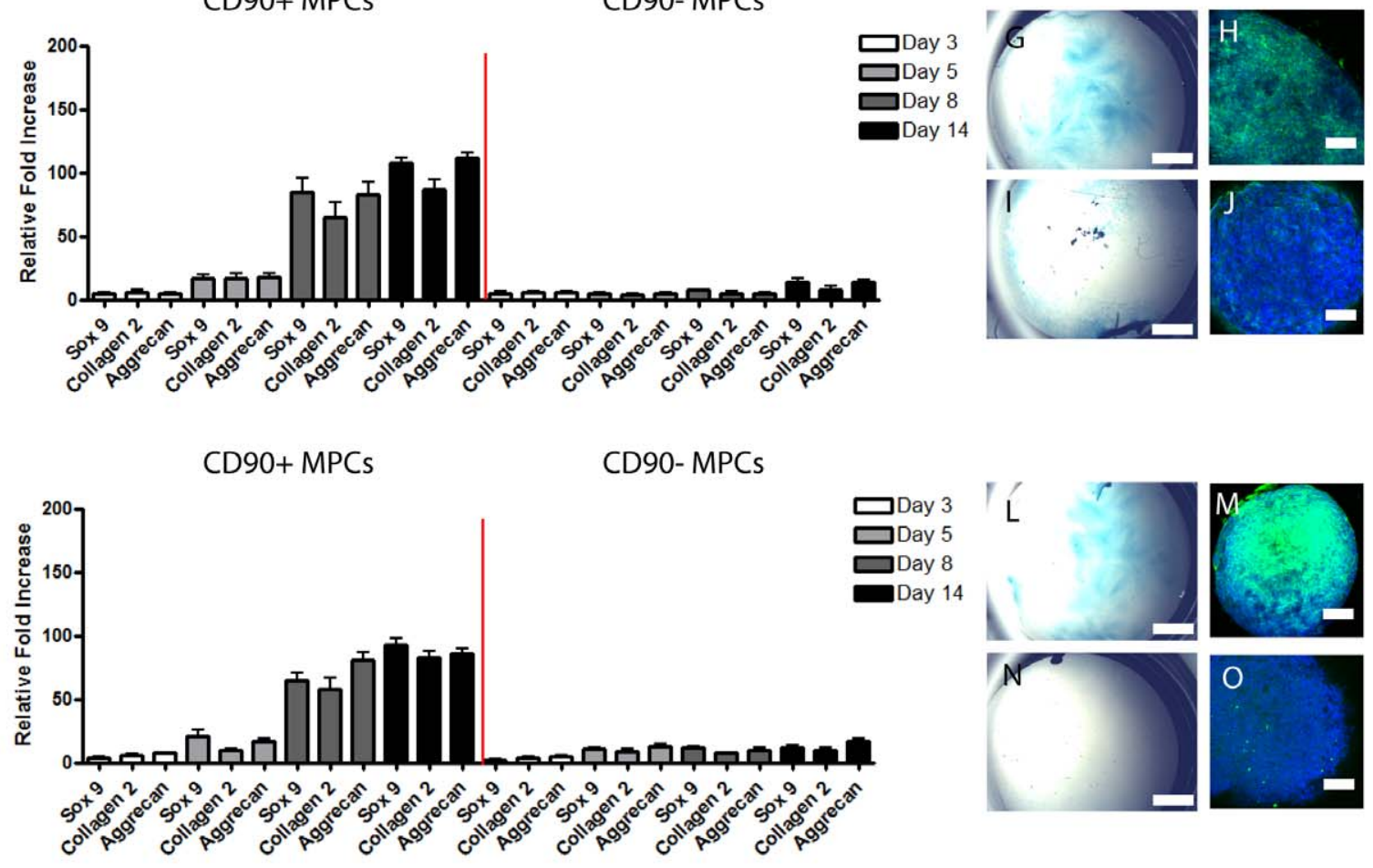

Normal canine
MPCs
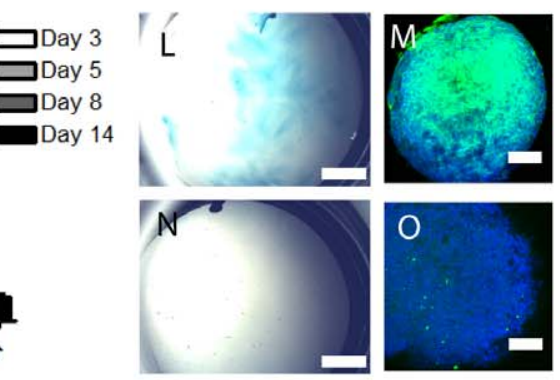

$\mathrm{P}$

CD90-MPCs
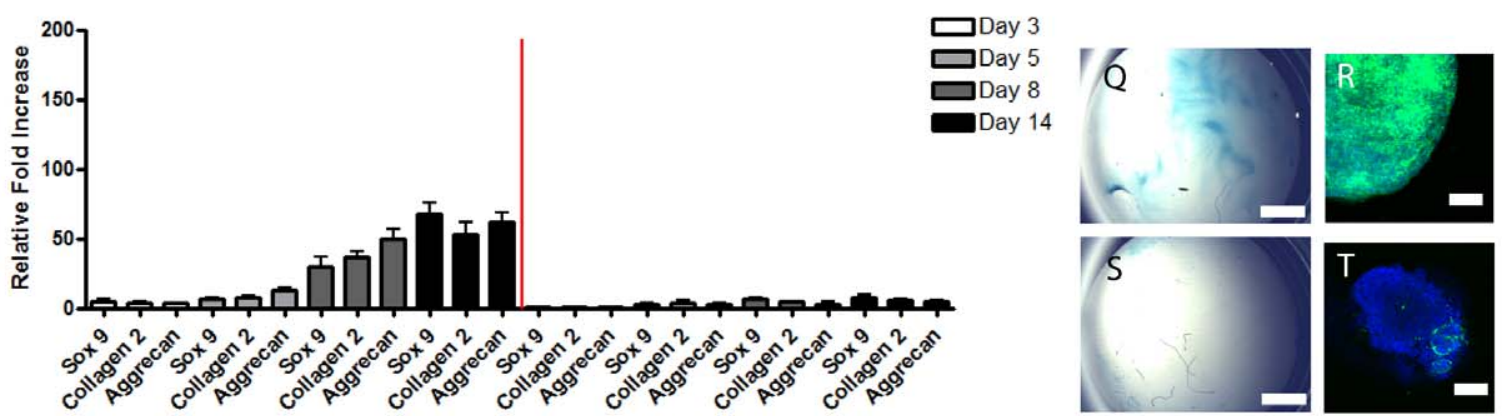

Figure 3. Multi-potent differentiation of CD90+/- sfMPCs. Normal and OA CD90+ or CD90 - sfMPCs were induced to differentiate into osteoblasts or adipocytes and stained with Akaline phosphatase (A) to indentify osteoblasts, or Oil Red-O (B) to identify lipid-containing cells (adipocytes). qRT-PCR was used to determine the relative level of adipocyte specific genes (Adiponectin, PPAR-gamma, [C]) and osteoblastic specific genes (Osteonectin, Sp7/Osterix [D]). ${ }^{*}=p>0.05$. doi:10.1371/journal.pone.0043616.g003 
A

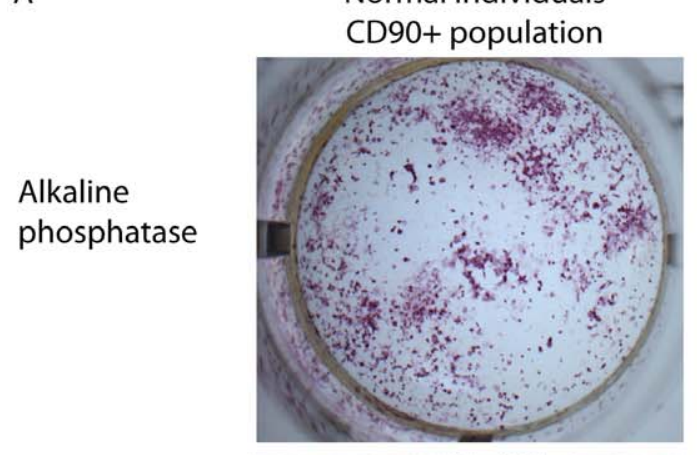

B

Oil Red-O
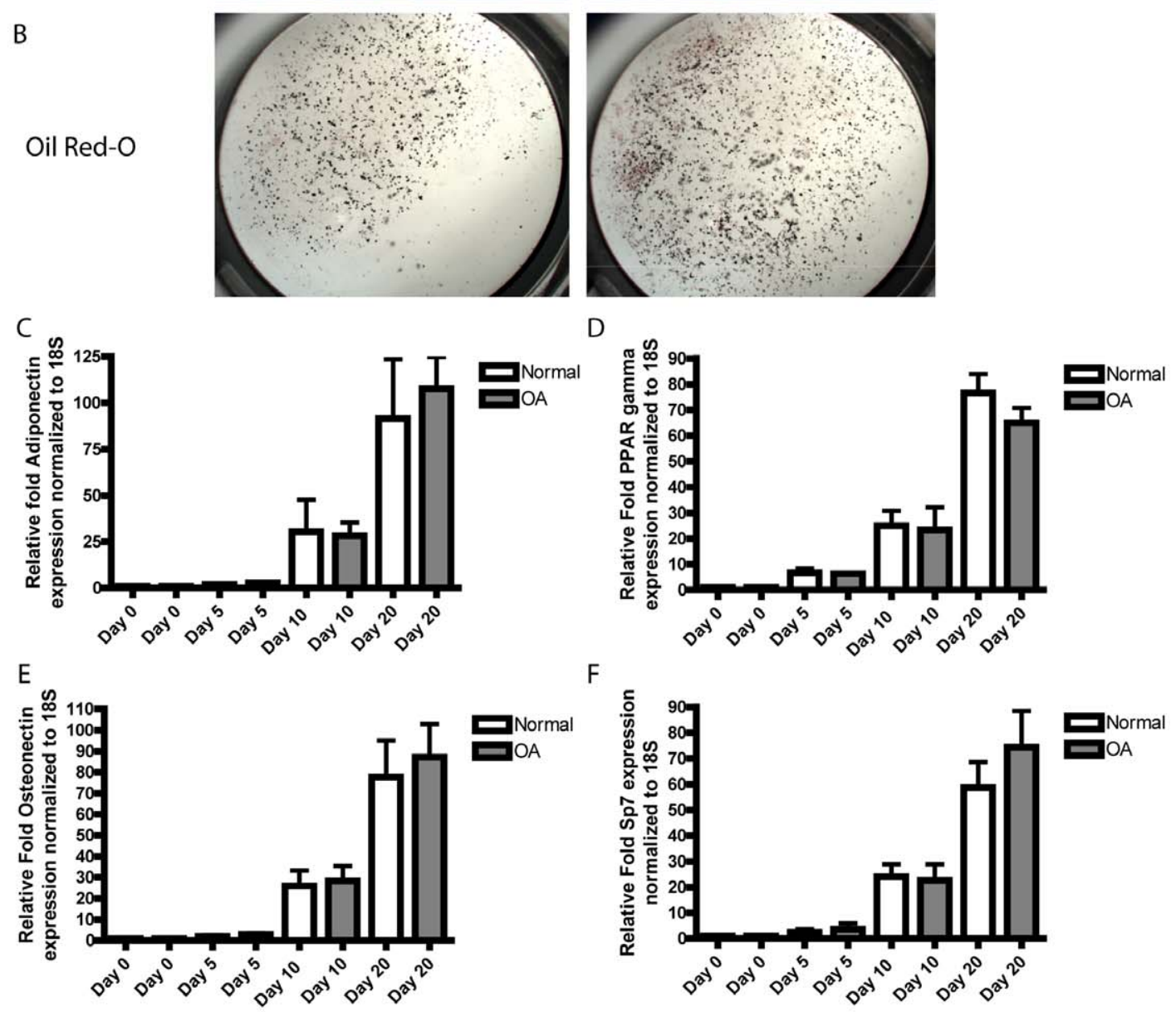

Figure 4. Aggregation and Chondrogenesis of sfMPCs. Normal and OA CD90+ sfMPCs were induced to differentiate without prior micro-mass aggregation. By day 14 normal sfMPCs expressed Sox9, Collagen2 and Aggrecan mRNA $(A, D)$ while OA sfMPCs expressed severely reduced levels $(A, D)$. Furthermore, normal sfMPCs generated a tissue-like structure that stained positive for Alcian Blue $(B, G)$, whereas OA sfMPCs did not $(C, F)$ and remained as a monolayer. Scale bars represent $200 \mu \mathrm{M}$. doi:10.1371/journal.pone.0043616.g004

were otherwise derived and cultured under identical conditions. Moreover, it would appear that this aggregation behaviour (which was consistent across the 5 human and 2 canine normal individuals tested) is lost within the OA phenotype (again consistent across the $5 \mathrm{OA}$ patients and 2 canines tested). We speculate that MPCs in the OA joint might be missing, or have masked, a cell surface receptor necessary for spontaneous aggregation. In continuing studies, we will examine if OA sfMPCs demonstrate any changes in gene expression compared to normal sfMPCs, specifically in genes related to pre-condensation (CGN-1, CNN-2) and/or within condensation (N-CAM, Tenascin G) [31].
To complement this line of investigation, micro-array studies comparing the global gene expression patterns of normal vs. OA sfMPCs have been completed and the data analysis is underway. Previously, studies have demonstrated that chondrocyte aggregation is mediated by $\beta 1$-Integrin [32], while Cadherins seem to play a dominant role in the aggregation of MSCs during chondrogenesis [33]. Therefore, the mechanism behind the failure of OA sfMPCs aggregation should be studied further to determine if genes expressed during the normal chondrogenic developmental process are aberrantly expressed. 

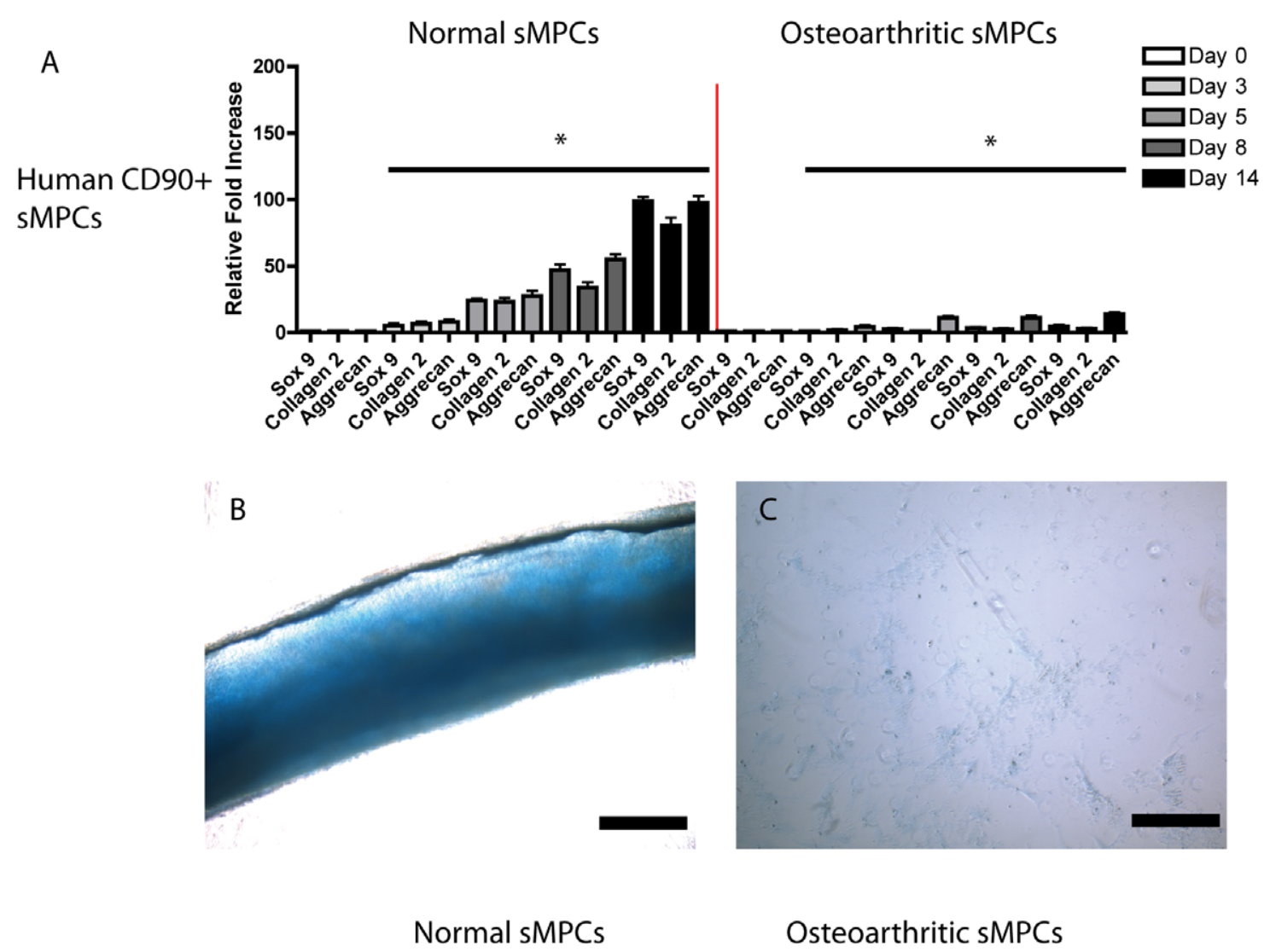

\section{Osteoarthritic SMPCs}
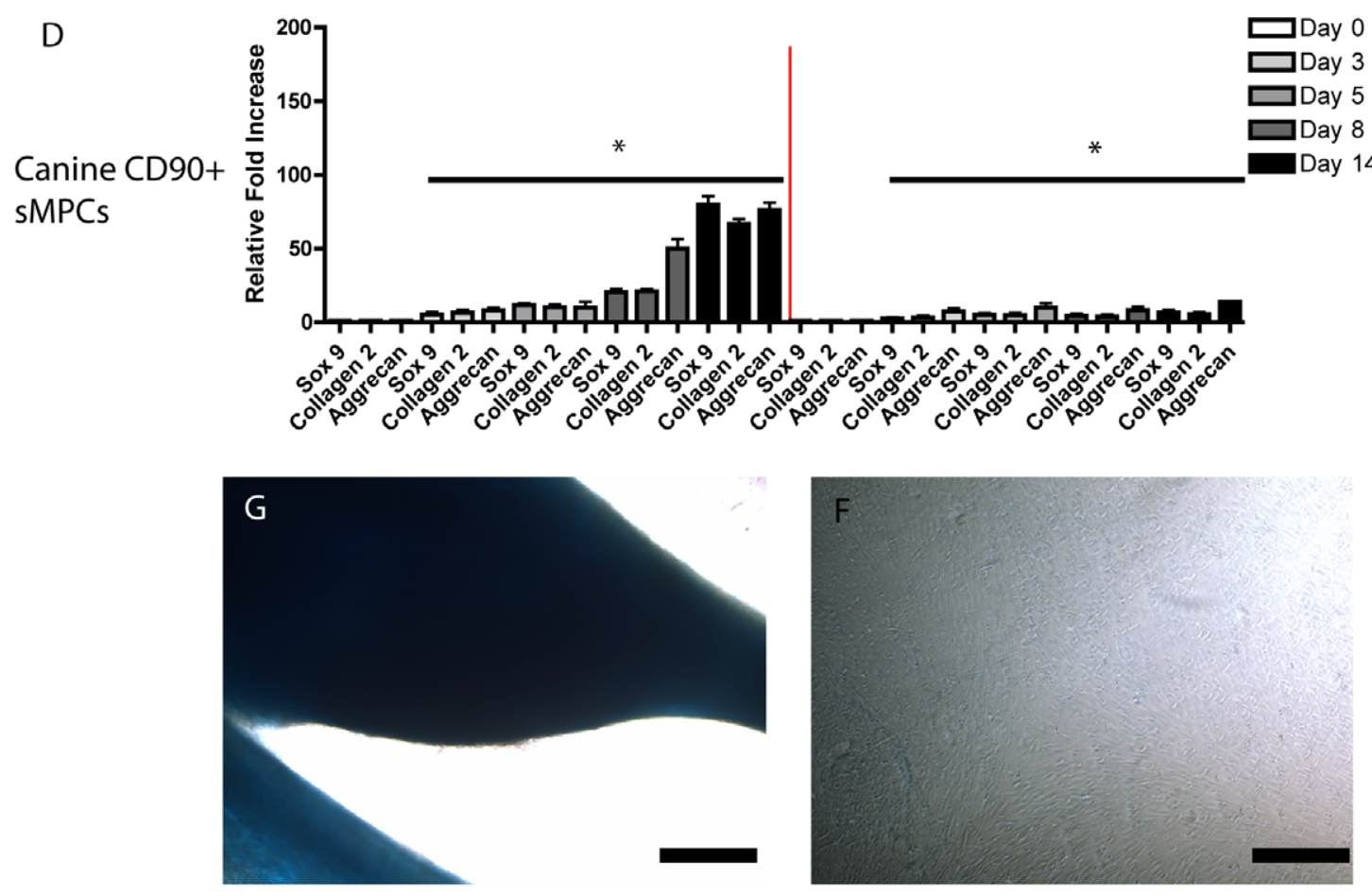

Figure 5. Staining and TEM of Derived Tissue from Normal sfMPCs. The resultant tissue-like masses were stained with antibodies to Collagen 2 (A) and Aggrecan (C), nuclei were stained with TOTO3 (B, D). Cells were found in closer association to each other (E), with higher magnification showing production of collagen fibres (F). Cells were also found in proximity with the produced collagen ECM (E, F). Scale bars represent $200 \mu \mathrm{M}$. doi:10.1371/journal.pone.0043616.g005

Since OA leads to a progressive loss of cartilage and synovial progenitors cells have the potential to contribute to articular cartilage repair in vivo [34], the inability of OA sfMPCs to spontaneously differentiate into chondrocytes suggests that cell-tocell aggregation and/or communication may be impaired in OA and somehow dampen the normal mechanism of chondrocyte 
replenishment from the synovium or synovial fluid. Should the cells of the synovium or synovial fluid be a reservoir of stem cells for normal articular cartilage maintenance and repair, these endogenous sources of chondro-biased cells would be a fundamental and new strategy for treating OA and cartilage injury if this loss of aggregation \& differentiation phenotype can be overcome.

\section{Methods}

\section{Ethics Statements}

Human: Informed consent to participate was obtained by written agreement. The study protocol was approved by the University of Calgary Research Ethics Board (Application number: 21987).

Canine: All procedures received approval by the institutional ethics board (University of Calgary, Animal Care Committee) and were carried out under the supervision of a veterinarian according to the guidelines of the Canadian Council on Animal Care.

\section{Human Subjects}

Patients with clinical and radiographic OA [20] with no other co-morbidities consented to arthrocentesis and had synovial fluid aspirated from their knees (mean volume $63.4 \mathrm{ml}+/-12.6 \mathrm{ml}$ ) during a visit to the University of Calgary Foothills Medical Clinic ( $N=5,3$ males age: 47, 50, 52; 2 females age 52, 54; Ethics ID \#21987). Synovial fluid from macroscopically normal knees was aspirated (mean volume $5.6 \mathrm{ml}+/-2.2 \mathrm{ml}$ ) from cadavers less than 4 hrs after death $(\mathrm{N}=5,2$ males age: 47,$54 ; 3$ female age 46 , 51, 52 Ethics ID \#21987). Tissue donors were received by the Southern Alberta Organ and Tissue Donation Program (SAOTDP), which obtains the medical history of every donor, including current medication, previous history of joint diseases, and other co-morbidities (e.g., cancer, diabetes, inflammatory diseases). All donor knees received $\mathrm{x}$-ray and macroscopic examination of the joint surfaces. Any abnormalities (cracking, blistering, darkening, abnormal wear) prompted exclusion from the study. In total seven cadaveric joints were examined and two were excluded from the study.

\section{Animals}

Animals were adult, outbred, skeletally mature canines ranging in age from 18-54 months. Briefly, unilateral cruciate ligament transection was performed through a lateral peripatellar athrot- omy ( $\mathrm{N}=2,1$ male, 1 female). Eight weeks after surgery, animals were euthanized, and sterile samples of joint fluid were withdrawn with a syringe. The volume of joint fluid from un-operated control knee joints ranged from 150-300 micro-liters; the volume of joint fluid from knees with cruciate transection ranged from 5-8 ml. Joint fluid was kept at $4{ }^{\circ} \mathrm{C}$ and transported to the laboratory within 2 hours of necropsy. All knee and hip joints were inspected for morphological abnormalities, which were not observed in any of the ipsilateral or contralateral hips, nor in any of the ipsilateral (control) knee joints.

\section{SMPC derivation and purification}

Human: Fresh synovial fluid was plated in untreated culture dishes and after $1-2 \mathrm{hrs}$ at $37^{\circ} \mathrm{C} / 5 \% \mathrm{CO}_{2}$ culture media was added. sMPG culture media consisted of DMEM (Invitrogen \# $11965), 10 \%$ FBS, $1 \%$ Pen/Strep, $1 \%$ Non-essential amino acids (NEAA), 0.2\% Beta-mercaptoethanol (BME) (all Invitrogen, Carlsbad, CA). Once cells had adhered to the plastic and reached 30-40\% confluence, the media was changed and the cells were allowed to reach $60-70 \%$ confluence. At this point the cells were dissociated and resuspended in Dulbecco's PBS (DPBS) at 1 million cells/ml. Primary labeled FACS antibodies (Table 1) to CD105, CD73, CD44, CD45, GD11b (All Becton, Dickinson and Company (BD), Franklin Lakes, NJ) were added to the suspension and the cells were sterilely sorted (University of Calgary, Flow Cytometry Core Facility). This CD105+, CD73+, CD44+, CD45-, CD11b- population accounted for $47.4 \%(+/-5.4 \%)$ of the total cells isolated from normal joints, and $67.2 \%(+/-3.1 \%)$ from joints with OA. Both normal and OA CD105+, CD73+, CD44+, CD45-, CD11b- cell populations were over $80 \%$ positive for CD90 before magnetic purification. The CD105+, CD73+, CD44+, CD45-, CD11b- cell population was returned to culture for 1 passage and then prepared for magnetic enrichment (an aliquot of this population was tested for multipotency, with differentiation into fat, bone and cartilage assayed with oil red, alizarin red and Alcian blue respectively). Purified sfMPCs were dissociated and treated with anti-CD90 (BD) and then a magnetically labeled secondary (BD), and subsequently exposed to a magnetic field for enrichment following the manufacturer's instructions. CD90-positive and CD90-negative fractions were cultured for one additional passage and then induced to differentiate. An aliquot of the cells at this passage were

Table 1. Primary antibodies used for analyses.

\begin{tabular}{|c|c|c|c|c|}
\hline Marker & Source & Identifies & Species & Cell target \\
\hline CD105 & Becton Dickinson & Endoglin & Human & MPC \\
\hline CD73 & Becton Dickinson & $5^{\prime}$-nucleotidase & Human & MPC \\
\hline CD44 & Becton Dickinson & CD44 & Human & MPC \\
\hline CD45 & Becton Dickinson & $\begin{array}{l}\text { Protein tyrosine phosphatase, } \\
\text { receptor type, C }\end{array}$ & Human & hematopoietic cells \\
\hline CD11b & Becton Dickinson & Integrin alpha $\mathrm{M}$ & Human & hematopoietic cells \\
\hline CD90 & Becton Dickinson & Thy-1 & Human/Canine & MPC \\
\hline CD34 & Becton Dickinson & CD34 & Canine & hematopoietic cells \\
\hline CD45 & Aviva Systems Biology & $\begin{array}{l}\text { Protein tyrosine phosphatase, } \\
\text { receptor type, C }\end{array}$ & Canine & hematopoietic cells \\
\hline Collagen 2 & Hybridoma Bank, lowa & Collagen 2 & Human/Canine & Chondrocytes \\
\hline Aggrecan & Santa Cruz & Aggrecan & Human/Canine & Chondrocytes \\
\hline
\end{tabular}


analyzed using FACS to confirm the presence or absence of CD105, CD90, CD73, GD44, CD45, CD11b (Figure 6).

Canine: Fresh synovial fluid was plated in untreated culture dishes and after $1-2 \mathrm{hrs}$ at $37^{\circ} \mathrm{C} / 5 \% \mathrm{CO}_{2}$ culture media was added. sMPC media (as above) was used. Once cells had adhered to the plastic and reached $30-40 \%$ confluence, the media was changed and the cells were allowed to reach $60-70 \%$ confluence. At this point the cells were dissociated and resuspended in Dulbecco's PBS (DPBS) at 1 million cells $/ \mathrm{ml}$. The total cell population was negatively purified using biotin-labeled antibodies to canine CD45 (Aviva Systems Biology, San Diego, CA) and CD34 (BD) following the manufacturer's instructions. This cell population was then selected for the CD90+ (BD) fraction using magnetic separation. CD90-positive and CD90-negative fractions were cultured for one additional passage and then induced to differentiate.

\section{Adipogenic Differentiation}

sfMPCs were plated in triplicate $(100,000$ cells/well/24 well dish) and exposed to adipogenic differentiation media for 20 days. Differentiation media consisted of sMPG culture media with $0.5 \mathrm{mM}$ isobutylmethylxanthine, $1 \mu \mathrm{M}$ dexamethasone, $10 \mu \mathrm{M}$ insulin, $200 \mu \mathrm{M}$ indomethacin (all Sigma). Media was changed every three days during the 20 day differentiation period.
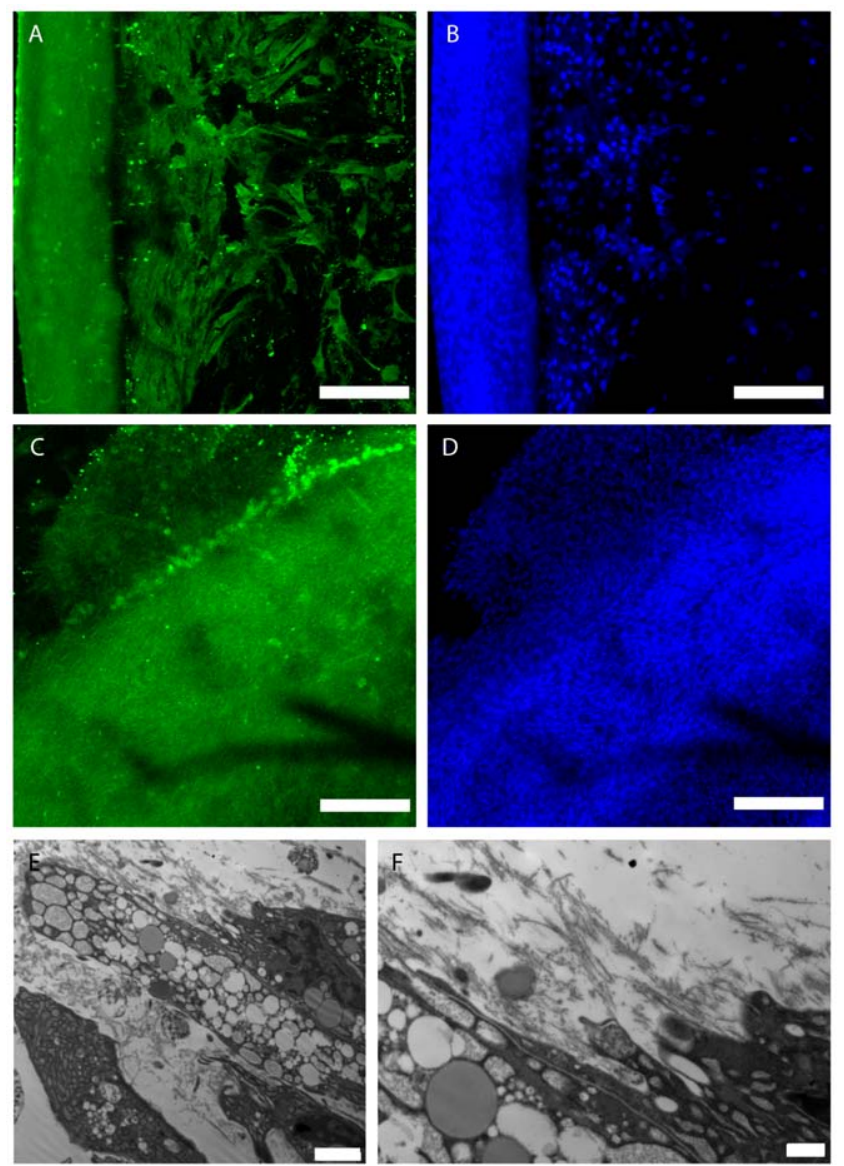

Figure 6. FACS characterization of human sfMPCs. Normal and $\mathrm{OA} C D 90+$ and $C D 90$ - subpopulations were assayed for expression of CD105, CD90, CD73, CD45 and CD11b prior to chondrogenic differentiation.

doi:10.1371/journal.pone.0043616.g006

\section{Osteogenic Differentiation}

sfMPCs were plated in triplicate $(100,000$ cells/well/24 well dish) and exposed to osteogenic differentiation media for 20 days. Differentiation media consisted of $\mathrm{sMPC}$ culture media with $0.1 \mu \mathrm{M}$ dexamethasone and $50 \mu \mathrm{M}$ ascorbate-2-phosphate. Media was changed every three days during the 20 day differentiation period.

\section{Chondrogenic Differentiation}

sfMPCs were plated in triplicate $(100,000$ cells/well/24 well dish) and exposed to chondrogenic media for 14 days with or without micro-mass aggregation. Aggregation was achieved by placing 100,000 cells in a $1.5 \mathrm{ml}$ sterile tube at $37^{\circ} \mathrm{C}$ overnight. Differentiation media consisted of $\mathrm{sMPC}$ culture media with 500 ng/mL BMP-2 (Peprotech, Rocky Hill, NJ), 10 ng/mL TGF$\beta 3$ (Peprotech), $10^{-8} \mathrm{M}$ dexamethasone (Sigma, St. Louis, MO), $50 \mu \mathrm{g} / \mathrm{mL}$ ascorbic acid (Sigma), $40 \mu \mathrm{g} / \mathrm{mL}$ proline (Invitrogen), $100 \mu \mathrm{g} / \mathrm{mL}$ pyruvate (Sigma) and supplemented with insulin, transferrin, and selenium (Sigma). Media was changed every three days during the 14-day differentiation period.

\section{Analysis of Differentiation}

Differentiation was assessed using quantitative RT-PCR (qRTPCR) and immunofluorescence (IF).

qRT-PCR: RNA was collected using Trizol (Invitrogen) and converted to cDNA using a High Capacity cDNA kit (Applied Biosystems (ABS), Carlsbad, CA). The cDNA was probed using pre-validated Taqman ${ }^{\mathrm{TM}}$ primer-sets for human Sox9, Collagen 2, Aggrecan, Osterix (SP7), Osteocalcin, PPAR-gamma, Adiponectin, and canine Sox9, Collagen 2 and Aggrecan (Table 2) on an ABI 7900HT using $18 \mathrm{~S}$ as the internal control and the ddCT method included within the ABI software to analyze results.

IF: Aggregates/tissues generated from sfMPCs were washed in PBS and fixed in $4 \%$ para-formaldehyde (PFA) in PBS at $4^{\circ} \mathrm{C}$ overnight. Aggregates were then permeabilized in $0.5 \%$ saponin (Sigma) in PBS at $4^{\circ} \mathrm{C}$ overnight, rinsed once in PBS, then blocked in $3 \% \mathrm{BSA}$ at $4^{\circ} \mathrm{C}$ overnight. Primary antibodies (Collagen 2: Developmental Studies Hybridoma Bank, Iowa. Aggrecan: Santa Cruz, Santa Cruz, CA) (Table 1) were diluted 1:50 in 3\% BSA, added to the cell samples and incubated overnight at $4{ }^{\circ} \mathrm{C}$. The aggregates were then washed 3 times with PBS and blocked again overnight at $4^{\circ} \mathrm{C}$. Following the block, the aggregates were incubated with an appropriate Alexa-fluor 488 secondary antibody (Molecular Probes, Carlsbad, CA) and Toto- $3^{\text {TM }}$ (Molecular Probes) overnight at $4{ }^{\circ} \mathrm{C}$. After incubation, the aggregates were washed thrice with PBS and mounted on slides with mountant (9:1 glycerol:PBS). Slides were analyzed using a Zeiss 510 confocal

Table 2. qRT-PCR probes used for analysis.

\begin{tabular}{lll}
\hline Marker & Source & Cell Target \\
\hline Sox 9 & $\mathrm{ABI}$ (human and canine probes) & Chondrocytes \\
Collagen 2 & $\mathrm{ABI}$ (human and canine probes) & Chondrocytes \\
Aggrecan & $\mathrm{ABI}$ (human and canine probes) & Chondrocytes \\
Osterix (SP7) & $\mathrm{ABI}$ (human) & Osteoblasts \\
Osteocalcin & $\mathrm{ABI}$ (human) & Osteoblasts \\
PPAR-gamma & $\mathrm{ABI}$ (human) & Adipocytes \\
Adiponectin & $\mathrm{ABI}$ (human) & Adipocytes \\
\hline doi:10.1371/journal.pone.0043616.t002 &
\end{tabular}


Microscope with 488, 568 and $633 \mathrm{~nm}$ filters. Images were prepared using Zeiss LSM image browsing software.

\section{Transmission Electron Microscopy}

For transmission electron microscopy (TEM), pre-cartilaginous tissue-like aggregates were collected after differentiation, and then washed in PBS before fixation with $4 \%$ glutaraldehyde in Millonig's phosphate buffer for 1 hour at room temperature. Post-fixation was carried out in $2 \% \mathrm{OsO}_{4}$ for 20 minutes. The tissues were then dehydrated in ethanol, and subsequently infiltrated with Polybed 812 resin (Polysciences). Polymerization was performed at $37^{\circ} \mathrm{C}$ for 24 hours. Silver-gray sections were cut with an ultramicrotome equipped with a diamond knife, and sections were stained with uranyl acetate and lead citrate, then examined in an H-7000 Hitachi electron microscope.

\section{References}

1. McGonagle D, Jones E (2008) A potential role for synovial fluid mesenchymal stem cells in ligament regeneration. Rheumatology (Oxford) 47:1114-6.

2. Jones EA, Crawford A, English A, Henshaw K, Mundy J, et al. (2008) Synovial fluid mesenchymal stem cells in health and early osteoarthritis: detection and functional evaluation at the single-cell level. Arthritis Rheum 58:1731-40.

3. Horie M, Sekiya I, Muneta T, Ichinose S, Matsumoto K, et al. (2009) Intraarticular Injected synovial stem cells differentiate into meniscal cells directly and promote meniscal regeneration without mobilization to distant organs in rat massive meniscal defect. Stem Cells 27:878-87.

4. Morito T, Muneta T, Hara K, Ju YJ, Mochizuki T, et al. (2008) Synovial fluidderived mesenchymal stem cells increase after intra-articular ligament injury in humans. Rheumatology (Oxford) 47:1137-43.

5. Koga H, Muneta T, Ju YJ, Nagase T, Nimura A, et al. (2007) Synovial stem cells are regionally specified according to local microenvironments after implantation for cartilage regeneration. Stem Cells 25:689-96.

6. Ando W, Tateishi K, Hart DA, Katakai D, Tanaka Y, et al. (2007) Cartilage repair using an in vitro generated scaffold-free tissue-engineered construct derived from porcine synovial mesenchymal stem cells. Biomaterials 28:546270 .

7. Ando W, Tateishi K, Katakai D, Hart DA, Higuchi C, et al. (2008) In vitro generation of a scaffold-free tissue-engineered construct (TEC) derived from human synovial mesenchymal stem cells: biological and mechanical properties and further chondrogenic potential. Tissue Eng Part A 14:2041-9.

8. Kanamoto T, Nakamura N, Nakata K, Yoshikawa H (2008) Articular cartilage regeneration using stem cells Clin Calcium 18:1744-9.

9. Hunziker EB, Rosenberg LC (1996) Repair of partial-thickness defects in articular cartilage: cell recruitment from the synovial membrane. J Bone Joint Surg Am 78:721-33.

10. Hunziker EB (2001) Growth-factor-induced healing of partial-thickness defects in adult rticular cartilage. Osteoarthritis Cartilage 9:22-32.

11. Koga H, Shimaya M, Muneta T, Nimura A, Morito T, et al. (2008) Local adherent technique for transplanting mesenchymal stem cells as a potential treatment of cartilage defect. Arthritis Res Ther 10:R84.

12. Koga H, Muneta T, Nagase T, Nimura A, Ju YJ, et al. (2008) Comparison of mesenchymal tissues-derived stem cells for in vivo chondrogenesis: suitable conditions for cell therapy of cartilage defects in rabbit. Cell Tissue Res 333:207-15.

13. Archer CW, Dowthwaite GP, Francis-West P (2003) Development of synovial joints. Birth Defects Res C Embryo Today 69:144-55.

14. Fan J, Varshney RR, Ren L, Cai D, Wang DA (2009) Synovium-derived mesenchymal stem cells: a new cell source for musculoskeletal regeneration. Tissue Eng Part B Rev 15:75-86.

15. Dominici M, Le BK, Mueller I, Slaper-Cortenbach I, Marini F, et al. (2006) Minimal criteria for defining multipotent mesenchymal stromal cells. The International Society for Cellular Therapy position statement. Cytotherapy 8:315-317.

16. Barker TH, Hagood JS (2009) Getting a grip on Thy-1 signaling. Biochim Biophys Acta 1793:921-3.

17. Mafi P, Hindocha S, Mafi R, Griffin M, Khan W (2011) Adult mesenchymal stem cells and cell surface characterization - a systematic review of the literature. Open Orthop J (Suppl 2):253-60.

\section{Statistical Analysis}

Each treatment (cell differentiation) and assay (IF, qRT-PCR) was performed in triplicate. Statistical analysis (ANOVA) was performed on qRT-PCR data using GraphPad Prism4 (GraphPad Software) and significance was set at $\mathrm{p}<0.05$.

\section{Acknowledgments}

We would like to thank Scott Ewald, Gary Rockl and the SAOTDP for assisting with the collection of cadaveric tissues.

\section{Author Contributions}

Conceived and designed the experiments: RJK JBR JRM DAH. Performed the experiments: RJK YEW JBR JRM. Analyzed the data: RJK. Contributed reagents/materials/analysis tools: LM JRM. Wrote the paper: RJK YEW.

18. Jones E, Churchman SM, English A, Buch MH, Horner EA, et al. (2010) Mesenchymal stem cells in rheumatoid synovium: enumeration and functional assessment in relation to synovial inflammation level. Ann Rheum Dis 69:450-7.

19. Nagase T, Muneta T, Ju YJ, Hara K, Morito T, et al. (2008) Analysis of the chondrogenic potential of human synovial stem cells according to harvest site and culture parameters in knees with medial compartment osteoarthritis. Arthritis Rheum 58:1389-98.

20. Altman R, Alarcón G, Appelrouth D, Bloch D, Borenstein D, et al. (1986) The American College of Rheumatology criteria for the classification and reporting of osteoarthritis of the knee. Arthritis Rheum 29:1039-49.

21. Koelling S, Miosge N (2009) Stem cell therapy for cartilage regeneration in osteoarthritis. Expert Opin Biol Ther 9:1399-405.

22. Han HS, Lee S, Kim JH, Seong SC, Lee MC (2010) Changes in chondrogenic phenotype and gene expression profiles associated with the in vitro expansion of human synovium-derived cells. J Orthop Res 28:1283-91.

23. Mackay AM, Beck SC, Murphy JM, Barry FP, Chichester CO, et al. (1998) Chondrogenic differentiation of cultured human mesenchymal stem cells from marrow. Tissue Eng 4:415-28.

24. Pittenger MF, Mackay AM, Beck SC, Jaiswal RK, Douglas R, et al. (1999) Multilineage potential of adult human mesenchymal stem cells. Science 284:143-7.

25. Luo W, Shitaye H, Friedman M, Bennett CN, Miller J, et al. (2008) Disruption of cell-matrix interactions by heparin enhances mesenchymal progenitor adipocyte differentiation. Exp Cell Res 314:3382-91.

26. Lim JJ, Scott L Jr, Temenoff JS (2011) Aggregation of bovine anterior cruciate ligament fibroblasts or marrow stromal cells promotes aggrecan production. Biotechnol Bioeng 108:151-62.

27. Okura H, Komoda H, Saga A, Kakuta-Yamamoto A, Hamada Y, et al. (2010) Properties of hepatocyte-like cell clusters from human adipose tissue-derived mesenchymal stem cells. Tissue Eng Part C Methods 16:761-70.

28. Wei C, Geras-Raaka E, Marcus-Samuels B, Oron Y, Gershengorn MC (2006) Trypsin and thrombin accelerate aggregation of human endocrine pancreas precursor cells. J Cell Physiol 206:322-8.

29. Gao F, Wu DQ, Hu YH, Jin GX, Li GD, et al. (2008) In vitro cultivation of isletlike cell clusters from human umbilical cord blood-derived mesenchymal stem cells. Transl Res 151:293-302.

30. Heng BC, Cowan CM, Basu S (2008) Temperature and calcium ions affect aggregation of mesenchymal stem cells in phosphate buffered saline. Cytotechnology 58:69-75.

31. Lorda-Diez CI, Montero JA, Diaz-Mendoza MJ, Garcia-Porrero JA, Hurle JM (2011) Defining the earliest transcriptional steps of chondrogenic progenitor specification during the formation of the digits in the embryonic limb. PLoS One 6:e24546.

32. Gigout A, Jolicoeur M, Nelea M, Raynal N, Farndale R, et al. (2008) Chondrocyte aggregation in suspension culture is GFOGER-GPP- and betal integrin-dependent. J Biol Chem 283:31522-30.

33. Bradley EW, Drissi MH (2011) Wnt5b regulates mesenchymal cell aggregation and chondrocyte differentiation through the planar cell polarity pathway. J Cell Physiol 226:1683-93.

34. Kurth TB, Dell'accio F, Crouch V, Augello A, Sharpe PT, et al. (2011) Functional mesenchymal stem cell niches in adult mouse knee joint synovium in vivo. Arthritis Rheum 63:1289-300. 